\title{
Simultaneous determination of main phenolic acids and flavonoids in tomato by micellar electrokinetic capillary electrophoresis
}

\section{Raúl Marti}

martir@uji.es

Mercedes

valcarce@uji.es

\section{José Manuel Herrero-Martínez}

jmherrer@uv.es

\section{Jaime Cebolla-Cornejo ${ }^{c,}$}

jaicecor@bct.upv.es

\section{Salvador Rosellóa, 1,*}

rosello@uji.es

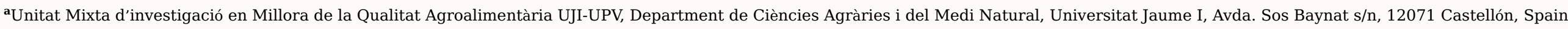

bepartamento de Química Analítica, Universitat de València, Dr. Moliner, 50, 46100 Burjassot, Spain

'Unitat Mixta d'investigació en Millora de la Qualitat Agroalimentària UJI-UPV, COMAV, Universitat Politècnica de València, Cno. de Vera, s.n., 46022 València, Spain

${ }^{*}$ Corresponding author.

${ }^{\mathbf{1} E q u a l}$ contribution.

\section{Abstract}

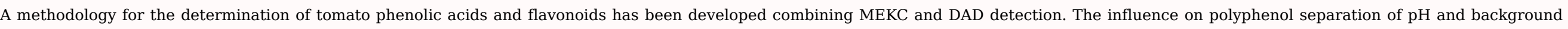

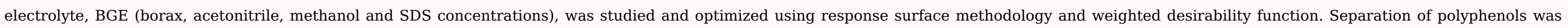

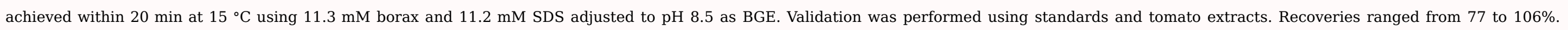

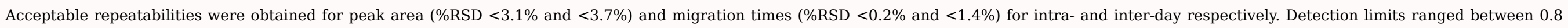

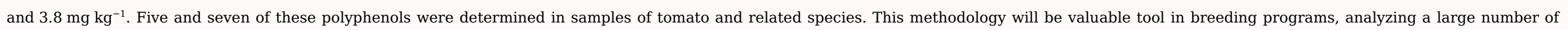
samples.

Keywords: Functional quality; MEKC; Plant breeding; Polyphenol; Response surface methodology

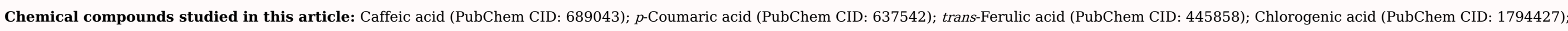
Kaempferol (PubChem CID: 5280863); Quercetin (PubChem CID: 5280343); Myricetin (PubChem CID: 5281672); Naringenin (PubChem CID: 932); Rutin (PubChem CID: 5280805)

\section{Introduction}

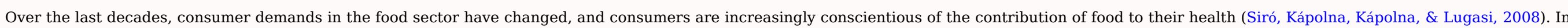




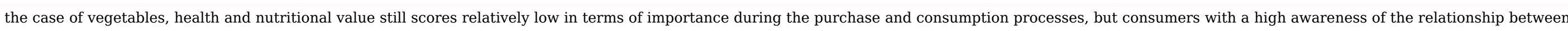

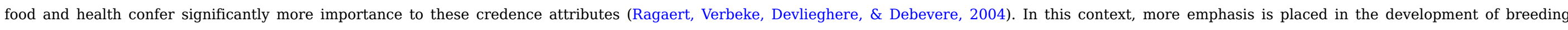
programs targeted to increase the concentration of bioactive compounds in vegetables (Goldman, 2011).

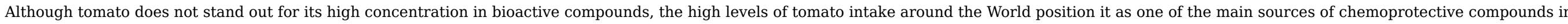

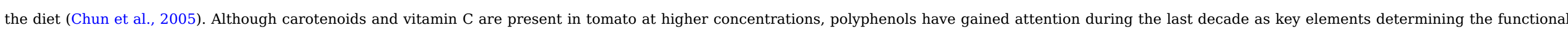

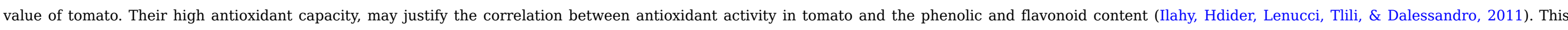

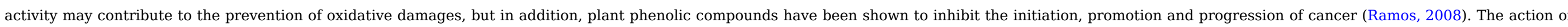

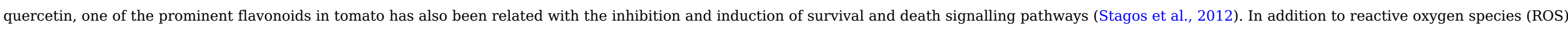

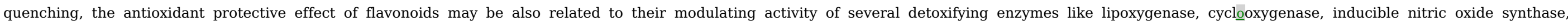
monooxygenase, xanthine oxidase and NADH oxidase which are involved in cancer development (reviewed by Gibellini et al. (2011))

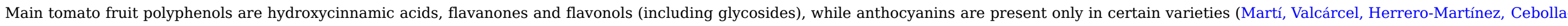

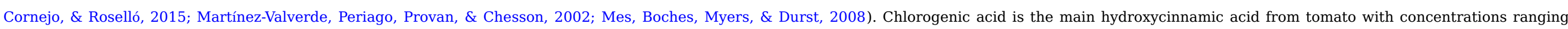

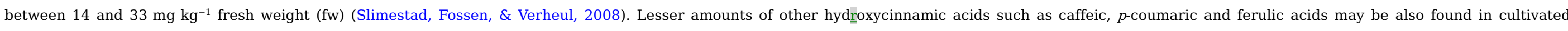

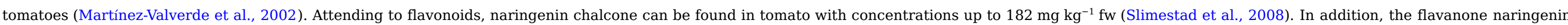

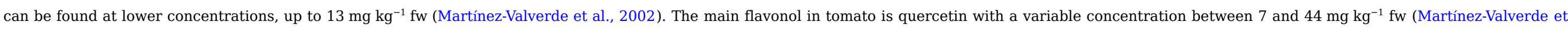

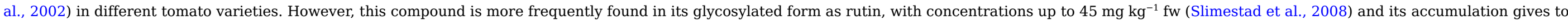

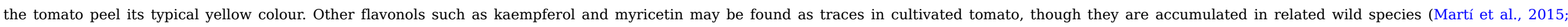
Martínez-Valverde et al., 2002; Shen, Chen, \& Wang, 2007).

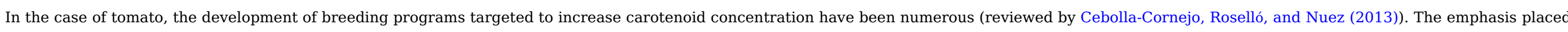

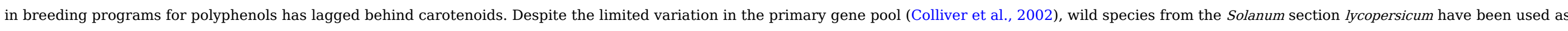
sources of variation. Following this approach, tomato flavonoid content has been increased using the wild tomato species Solanum pennellii Correl (Willits et al., 2005).

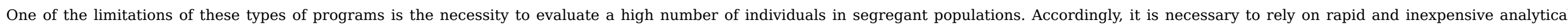

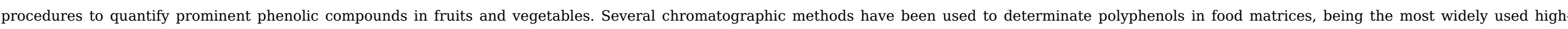

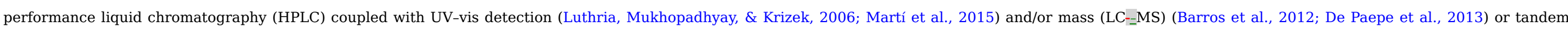

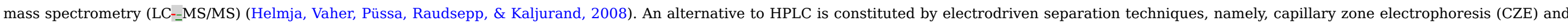

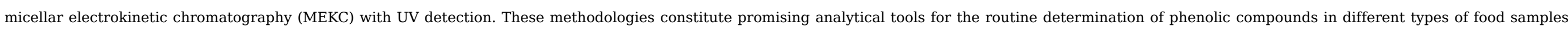

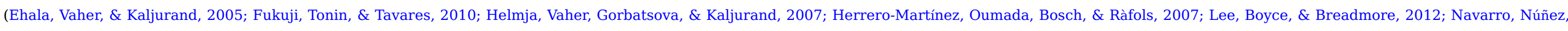

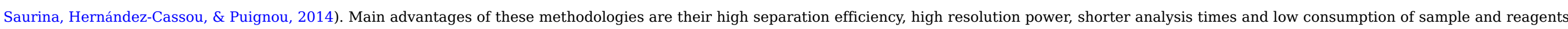

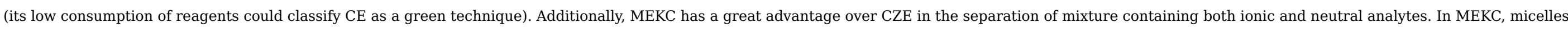

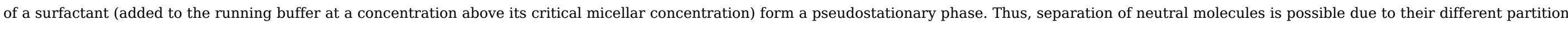

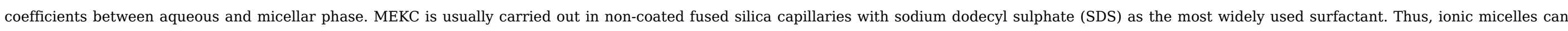

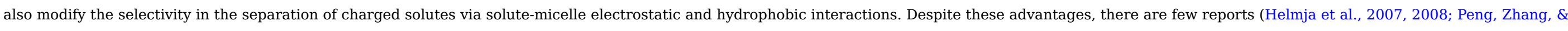
Ye, 2008) dealing with the determination of phenolic compounds in tomato by these techniques.

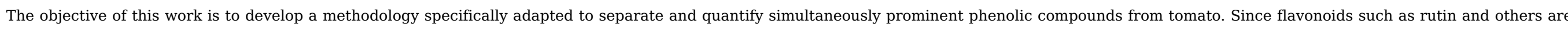

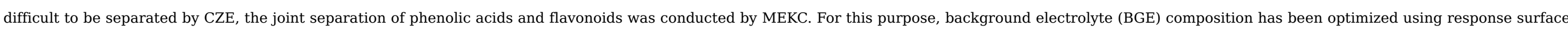
methodology (RSM) to obtain the optimum separation conditions. The optimized methodology was successfully validated and applied to the analysis of different tomato samples. 


\section{Material and methods}

\subsection{Chemicals and reagents}

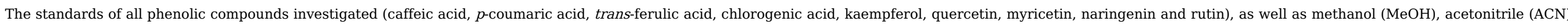

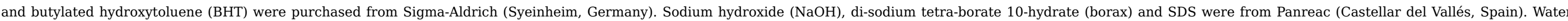

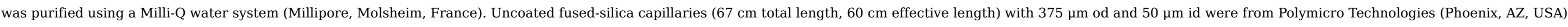

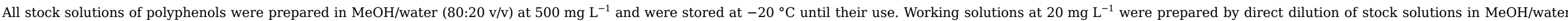
$(48: 52 \mathrm{v} / \mathrm{v})$. All solutions were filtered through a $0.2 \mu \mathrm{m}$ pore diameter PTFE filter before to be used.

\subsection{Instrumentation and conditions}

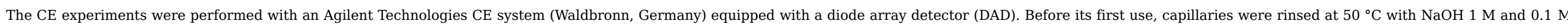

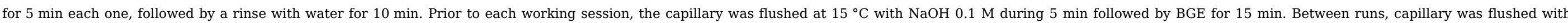

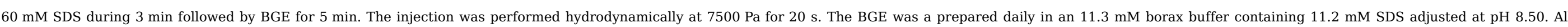

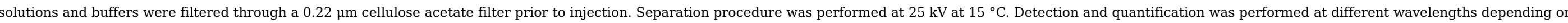

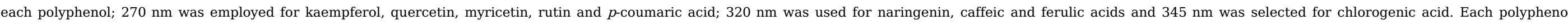
spectrum was recorded using a DAD detector for later identification of compounds.

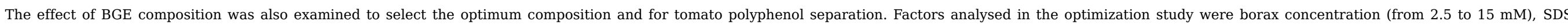

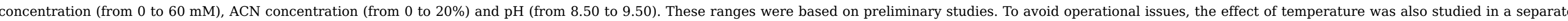

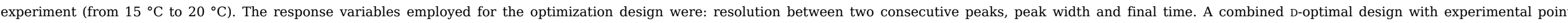

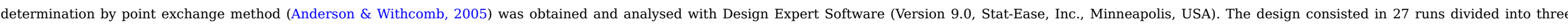

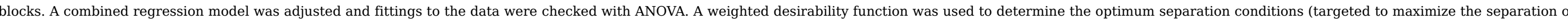

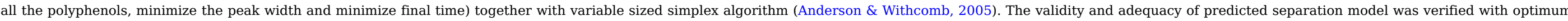
separation conditions (five replicates) comparing predictions with observed values using a two sided $t$-test $(\alpha=0.05)$

\subsection{Plant material and sample extraction}

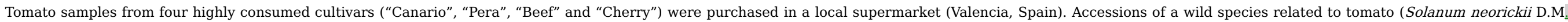

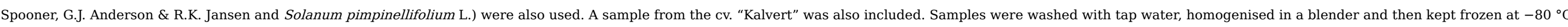

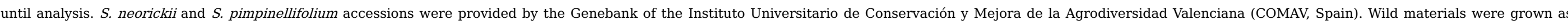

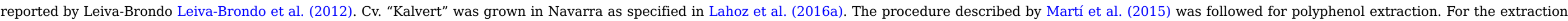

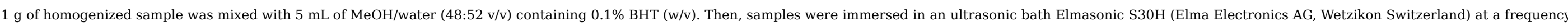

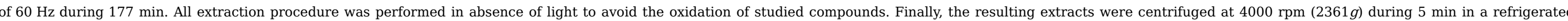
centrifuge at $4{ }^{\circ} \mathrm{C}$, and supernatants were filtered through a $0.2 \mu \mathrm{m}$ pore size PTFE filter before their injection in the CE system.

\subsection{Method validation}

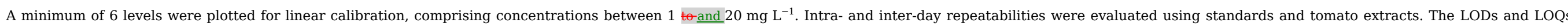
were calculated for polyphenols present in tomato as the lowest concentration that provides a signal to noise ratio (S/N) equal to 3 and 10 , respectively.

Real samples were also analysed using HPLC in order to provide a comparison with alternative methodology. For this purpose, the method and equipment described by Martí et al. (2015) was used.

\section{Results and discussion}




\subsection{Optimization of MEKC conditions}

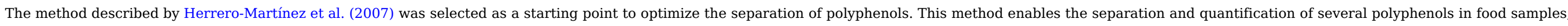

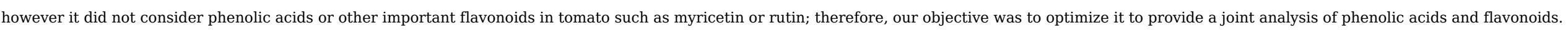

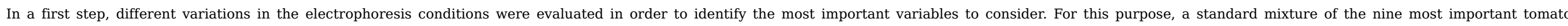
polyphenols (20 $\mathrm{mg} \mathrm{L}^{-1}$ ) was used, including caffeic, $p$-coumaric, ferulic and chlorogenic acids, kaempferol, quercetin, myricetin, naringenin and rutin.

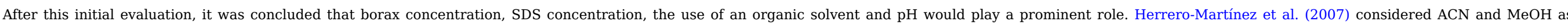

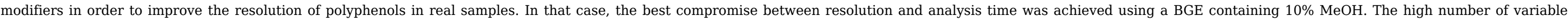

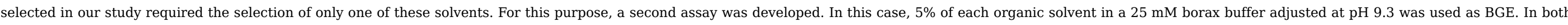

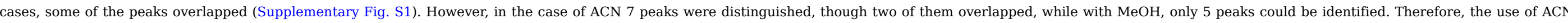
provided a better resolution than $\mathrm{MeOH}$ of the problematic peaks, and it was selected as organic modifier for further optimization.

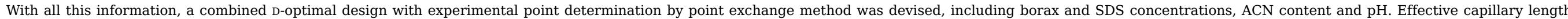
$(60 \mathrm{~cm})$ and separation temperature $\left(20^{\circ} \mathrm{C}\right)$ were kept as fixed factors.

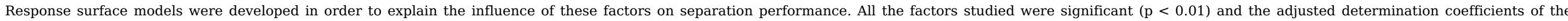

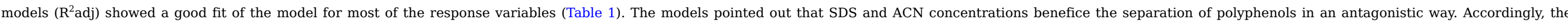

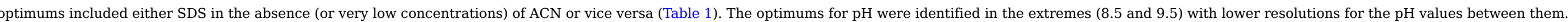

Table 1 Results from the optimization of BGE composition using RSM. Goodness of fit, significant factors identified, and best factor combinations are indicated.

\begin{tabular}{|c|c|c|c|c|c|c|c|c|c|c|c|c|c|c|c|c|c|c|c|}
\hline & & \multirow[t]{2}{*}{ Final time } & \multicolumn{8}{|c|}{ Peak resolution } & \multicolumn{9}{|c|}{ Peak width } \\
\hline & & & $1-2^{\mathrm{a}}$ & $2-3$ & $3-4$ & $4-5$ & $5-6$ & $6-7$ & $7-8$ & $8-9$ & 1 & 2 & 3 & 4 & 5 & 6 & 7 & 8 & 9 \\
\hline \multicolumn{2}{|c|}{ Model R²adjusted } & 0.96 & 0.08 & 0.55 & 0.20 & 0.05 & 0.10 & 0.78 & 0.97 & 0.60 & 0.67 & 0.65 & 0.70 & 0.63 & 0.86 & 0.89 & 0.64 & 0.81 & 0.72 \\
\hline \multicolumn{2}{|c|}{$\begin{array}{l}\text { Significant factors and interactions } \\
\text { in the RSM models }\end{array}$} & $\begin{array}{l}\text { A, B, C, } \\
A B, A C, A^{2}\end{array}$ & $\mathrm{ND}^{\mathrm{c}}$ & $\begin{array}{l}\mathrm{C}, \\
\mathrm{AC}, \\
\mathrm{BD}\end{array}$ & ND & ND & ND & $\begin{array}{l}\mathrm{A}, \mathrm{AB}, \\
\mathrm{AC}, \mathrm{A}^{2}, \\
\mathrm{C}^{2}\end{array}$ & $\begin{array}{l}\mathrm{A}, \mathrm{C}, \mathrm{D}, \mathrm{AB}, \mathrm{AD} \\
\mathrm{BC}, \mathrm{CD}, \mathrm{A}^{2}, \mathrm{~B}^{2}, \mathrm{C}^{2}\end{array}$ & $\begin{array}{l}\mathrm{B}, \mathrm{AB} \\
\mathrm{BC}, \mathrm{CD}, \\
\mathrm{A}^{2}\end{array}$ & $\begin{array}{l}\text { A, } \\
\text { B, C }\end{array}$ & $\begin{array}{l}\text { A, B, C, } \\
\mathrm{AB}, \mathrm{BC}\end{array}$ & $\begin{array}{l}\text { A, B, C, } \\
\text { D, BC }\end{array}$ & B, C & $\begin{array}{l}\text { A, B, } \\
\text { C, } \\
\text { BC }\end{array}$ & $\begin{array}{l}A, B, C \\
A B, B C \\
C D\end{array}$ & $\begin{array}{l}\mathrm{B}, \mathrm{AB} \\
\mathrm{BC}, \mathrm{CD}\end{array}$ & $\begin{array}{l}\mathrm{B}, \mathrm{BC} \\
\mathrm{CD}, \mathrm{C}^{2}\end{array}$ & $\begin{array}{l}\mathrm{B}, \mathrm{D}, \mathrm{AB}, \mathrm{BC} \\
\mathrm{BD}, \mathrm{CD}, \mathrm{A}^{2}, \mathrm{D}^{2}\end{array}$ \\
\hline \multirow[t]{4}{*}{ Best factor combination } & $\mathrm{ACN}$ & 0.0 & 20.0 & 20.0 & 0.0 & 0.0 & ND & 0.0 & 7.3 & 0.0 & 0.0 & 0.0 & 0.0 & 0.0 & 0.0 & 0.0 & 0.0 & 0.0 & 20.0 \\
\hline & $\mathrm{pH}$ & 9.5 & ND & 8.5 & 8.5 & 8.5 & ND & 9.3 & 8.5 & 8.5 & 8.5 & 8.5 & 8.5 & 8.5 & 8.5 & 8.5 & 8.5 & 8.5 & 9.5 \\
\hline & SDS & 0.0 & ND & 0.0 & 0.0 & 0.0 & 0.0 & 0.0 & 35.0 & 60.0 & 0.0 & 57.0 & 0.0 & 0.0 & 0.0 & 5.0 & 3.0 & 30.0 & 33.0 \\
\hline & Borax & 2.5 & 15.0 & 15.7 & ND & 15.0 & ND & 15.0 & 6.0 & 6.8 & 3.3 & 2.5 & 14.5 & 5.0 & 6.3 & 0.0 & 15.0 & 7.5 & 10.0 \\
\hline
\end{tabular}

a Peak identification: $1=$ rutin, $2=$ naringenin, $3=$ ferulic acid, $4=p$-coumaric acid, $5=$ chlorogenic acid, $6=$ kaempferol, $7=$ myricetin, $8=$ quercetin, $9=$ caffeic acid.

b Model factors: $\mathrm{A}=\operatorname{borax}(\mathrm{mM}), \mathrm{B}=\mathrm{SDS}(\mathrm{mM}), \mathrm{C}=\mathrm{ACN}(\%), \mathrm{D}=\mathrm{pH}$.

${ }^{\mathbf{c}} \mathrm{ND}=$ Not dependent.

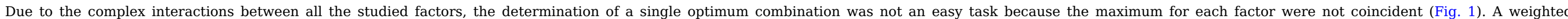

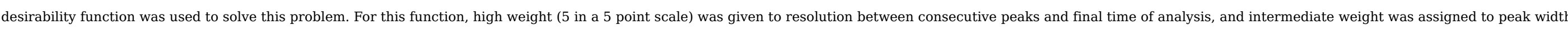

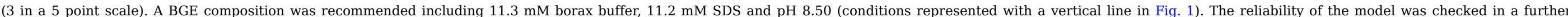




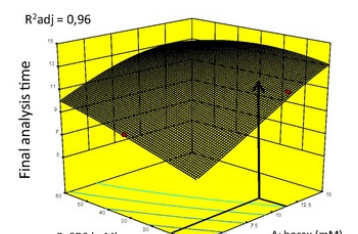

B: $\operatorname{sos}($

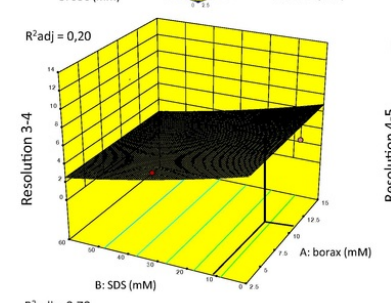

: $: 50$
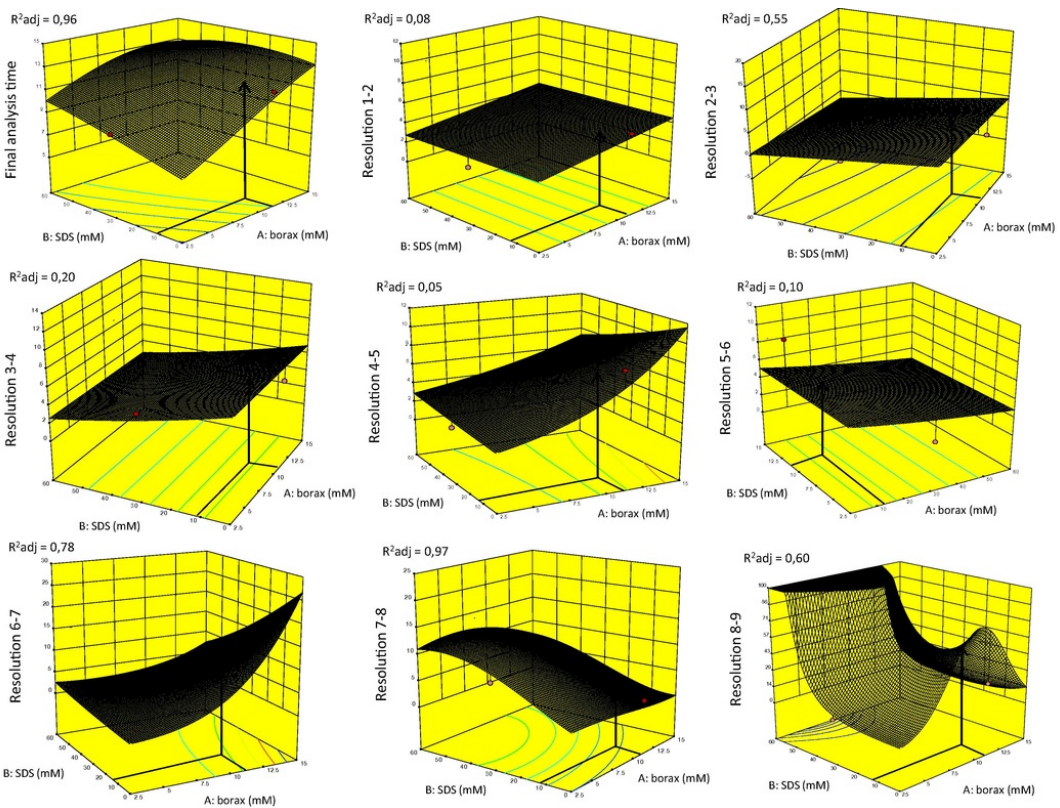

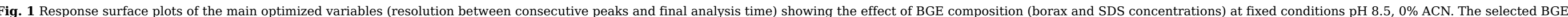

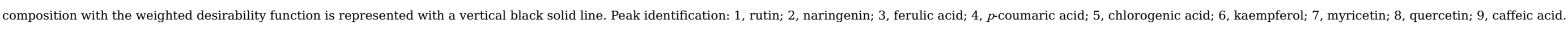

Table 2 Results of predicted (mean \pm SD), limits of 95\% prediction interval and the real values (mean \pm SD) for all optimized response factors.

\begin{tabular}{|c|c|c|c|c|}
\hline & Optimized parameter & Predicted & 95\% Prediction interval & Experimental \\
\hline \multicolumn{2}{|r|}{ Final analysis time } & $10.3 \pm 0.7$ & $8.6-12.6$ & $11.1 \pm 0.2$ \\
\hline \multirow[t]{8}{*}{ Resolution between } & Rutin-naringenin & $3.6 \pm 2.3$ & $0-8.6$ & $2.3 \pm 0.1$ \\
\hline & Naringenin-ferulic acid & $4.4 \pm 3.3$ & $0-12.7$ & $4.8 \pm 0.2$ \\
\hline & Ferulic acid- $p$-coumaric acid & $6.1 \pm 2.8$ & $1.3-13.4$ & $8.1 \pm 0.1$ \\
\hline & $p$-Coumaric acid-chlorogenic acid & $4.2 \pm 2.0$ & $1.0-13.5$ & $7.7 \pm 0.1$ \\
\hline & Chlorogenic acid-kaempferol & $4.7 \pm 2.7$ & $1.0-15.2$ & $6.1 \pm 0.2$ \\
\hline & Kaempferol-myricetin & $2.0 \pm 1.5$ & $0-7.8$ & $2.7 \pm 0.1$ \\
\hline & Myricetin-quercetin & $3.7 \pm 1.1$ & $1.3-7.3$ & $2.8 \pm 0.1$ \\
\hline & Quercetin-caffeic acid & $44.1 \pm 48.6$ & $0.1-2223.8$ & $22.3 \pm 1.3$ \\
\hline \multirow[t]{3}{*}{ Peak width } & Rutin & $0.050 \pm 0.014$ & $0.024-0.094$ & $0.054 \pm 0.001$ \\
\hline & Naringenin & $0.063 \pm 0.026$ & $0-0.130$ & $0.044 \pm 0.001$ \\
\hline & Ferulic acid & $0.057 \pm 0.017$ & $0.025-0.117$ & $0.043 \pm 0.001$ \\
\hline
\end{tabular}




\begin{tabular}{|l|l|l|l|l}
\hline$p$-Coumaric acid & $0.062 \pm 0.016$ & $0.035-0.118$ & $0.043 \pm 0.001$ \\
\hline Chlorogenic acid & $0.056 \pm 0.012$ & $0.032-0.093$ & $0.058 \pm 0.001$ \\
\hline Kaempferol & $0.072 \pm 0.014$ & $0.043-0.115$ & $0.055 \pm 0.001$ \\
\hline Myricetin & $0.113 \pm 0.037$ & $0.051-0.312$ & $0.063 \pm 0.002$ \\
\hline Quercetin & $0.131 \pm 0.043$ & $0.057-0.419$ & $0.104 \pm 0.005$ \\
\hline Caffeic acid & $0.098 \pm 0.217$ & $0-0.700$ & $0.072 \pm 0.001$ \\
\hline
\end{tabular}

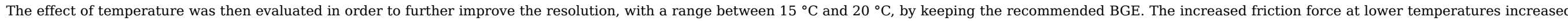
the time for analysis, but also improved the separation of close peaks (Fig. 2A). Once optimized the conditions for the analysis, the resulting MEKC method was validated.

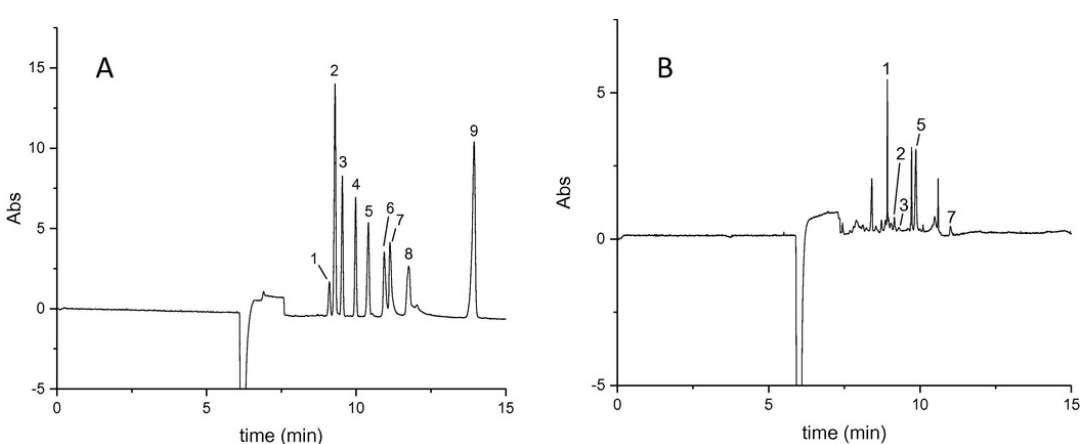

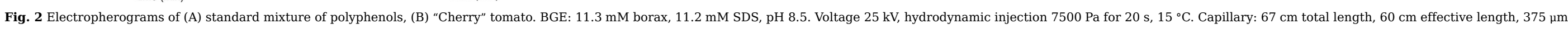
od and $50 \mu \mathrm{m}$ id. Peak identification: 1, rutin; 2, naringenin; 3, ferulic acid; 4, p-coumaric acid; 5, chlorogenic acid; 6, kaempferol; 7, myricetin; 8, quercetin; 9, caffeic acid.

\subsection{Validation of MEKC method}

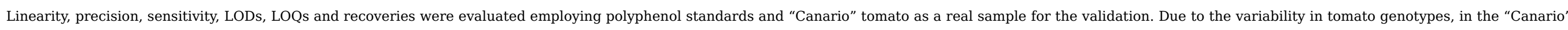
sample used for validation not all the polyphenols were present and \%RSD was calculated with fortified samples.

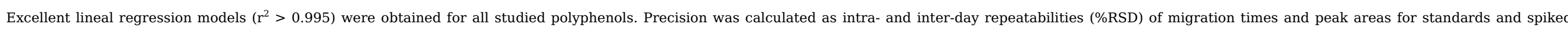

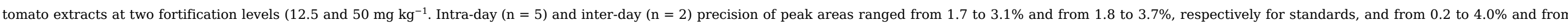

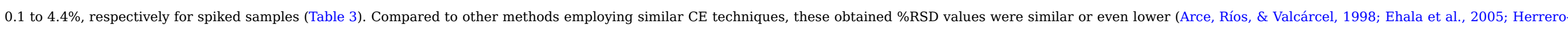

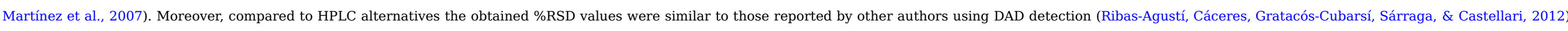

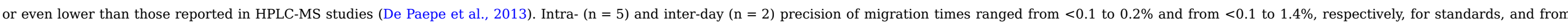
$<0.1$ to $0.2 \%$ and from 0.3 to $1.7 \%$ respectively for spiked samples (Table 3). These values are similar to those reported by other authors (De Paepe et al., 2013).

Table 3 Analytical performance parameters of the optimized method using polyphenol standards and spiked tomato samples.

\begin{tabular}{|c|c|c|c|c|c|c|c|c|c|c|}
\hline \multirow[t]{2}{*}{ Compounds } & \multicolumn{3}{|c|}{$\begin{array}{l}\text { Calibration equation } \\
\qquad y=a x+b\end{array}$} & \multicolumn{2}{|c|}{$\begin{array}{l}\text { Intra-day repeatability }^{\mathrm{a}} \\
\quad(\mathrm{n}=5) \mathrm{RSD}(\%)\end{array}$} & \multicolumn{2}{|c|}{$\begin{array}{l}\text { Inter-day repeatability }^{\mathrm{a}} \\
\quad(\mathrm{n}=2) \mathrm{RSD}(\%)\end{array}$} & \multirow[t]{2}{*}{$\begin{array}{c}\text { LOD; LOQ } \\
\left(\mathrm{mg} \mathrm{kg}^{-1}\right)\end{array}$} & \multicolumn{2}{|c|}{$\begin{array}{l}\text { Tomato samples, } \\
\text { Recovery (\%), } \mathrm{n}=5\end{array}$} \\
\hline & $\mathrm{a}$ & $\mathrm{b}$ & $\mathrm{r}^{2}$ & $\mathrm{t}_{\mathrm{m}}$ & Area & $t_{m}$ & Area & & $\begin{array}{c}\text { Low } \\
\left(12.5 \mathrm{mg} \mathrm{kg}^{-1}\right)\end{array}$ & $\begin{array}{c}\text { High } \\
\left(50 \mathrm{mg} \mathrm{kg}^{-1}\right)\end{array}$ \\
\hline Caffeic acid & 6.145 & -2.403 & 0.9971 & $0.1 ; 0.2$ & $2.3 ; 1.3$ & $0.1 ; 0.9$ & $2.9 ; 3.6$ & $3.1 ; 10.3$ & $106 \pm 2$ & $93 \pm 1$ \\
\hline
\end{tabular}




\begin{tabular}{|c|c|c|c|c|c|c|c|c|c|c|}
\hline$p$-Coumaric acid & 3.988 & -1.545 & 0.9950 & $0.1 ;<0.1$ & $2.5 ; 0.8$ & $0.6 ; 1.7$ & 3.6; 0.1 & $1.6 ; 5.2$ & $85 \pm 4$ & $98 \pm 1$ \\
\hline Ferulic acid & 2.526 & -0.602 & 0.9961 & $0.1 ;<0.1$ & $2.4 ; 1.5$ & $0.2 ; 0.3$ & $3.7 ; 1.0$ & $1.3 ; 4.3$ & $101 \pm 1$ & $103 \pm 2$ \\
\hline Chlorogenic acid & 3.378 & -1.015 & 0.9971 & $<0.1 ; 0.1$ & $3.1 ; 0.6$ & $0.2 ; 0.7$ & $3.7 ; 1.2$ & $3.8 ; 12.6$ & $101 \pm 2$ & $93 \pm 1$ \\
\hline Kaempferol & 3.039 & -2.614 & 0.9958 & $0.1 ; 0.1$ & $2.5 ; 3.5$ & $0.3 ; 0.9$ & $1.8 ; 4.4$ & $3.1 ; 10.2$ & $103 \pm 5$ & $95 \pm 4$ \\
\hline Quercetin & 3.241 & -0.403 & 0.9985 & $<0.1 ; 0.1$ & $2.3 ; 1.7$ & $<0.1 ; 0.3$ & $1.9 ; 3.5$ & $3.3 ; 10.9$ & $95 \pm 3$ & $86 \pm 1$ \\
\hline Myricetin & 4.236 & -2.546 & 0.9978 & $0.1 ; 0.1$ & $1.8 ; 1.4$ & $<0.1 ; 0.6$ & $2.9 ; 2.4$ & $2.5 ; 8.2$ & $96 \pm 1$ & $83 \pm 1$ \\
\hline Naringenin & 4.687 & -0.388 & 0.9977 & $0.2 ; 0.1$ & $2.4 ; 4.0$ & $1.4 ; 0.6$ & $2.2 ; 3.3$ & $0.8 ; 2.6$ & $77 \pm 5$ & $77 \pm 4$ \\
\hline Rutin & 1.703 & -0.116 & 0.9986 & $0.1 ;<0.1$ & $1.7 ; 0.2$ & $0.4 ; 0.3$ & $2.9 ; 1.9$ & $3.3 ; 11.1$ & $96 \pm 6$ & $89 \pm 0$ \\
\hline
\end{tabular}

a RSD obtained with: standards; tomato extracts.

b Detection and quantification limits obtained with tomato extracts.

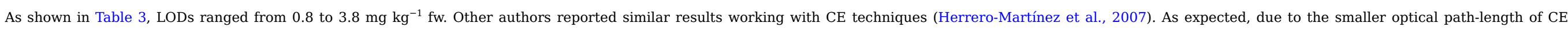
detection cell compared to HPLC, LODs obtained are higher than those reported by other authors working with HPLC with DAD detection (Ribas-Agustí et al., 2012).

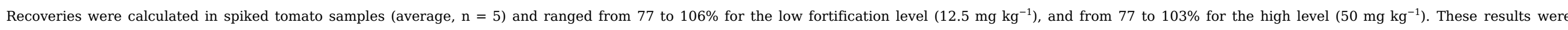
comparable or even better than those reported by other authors (De Paepe et al., 2013; Ribas-Agustí et al., 2012; Sakakibara, Honda, Nakagawa, Ashida, \& Kanazawa, 2003).

\subsection{Quantitation studies and application to real samples}

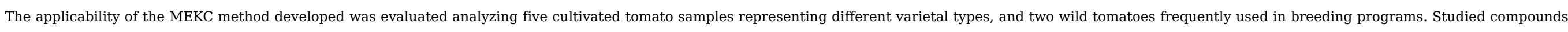

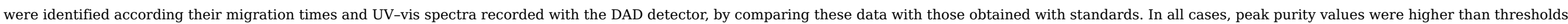

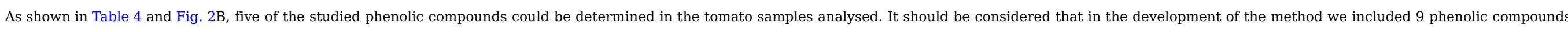
described at any time in tomato, but the occurrence of some of them is very rare or are present as traces.

Table 4 Determination of main polyphenols $\left(\mathrm{mg} \mathrm{kg}^{-1} \mathrm{fw}\right)$ in tomato and tomato wild relatives (mean $\left.\pm \mathrm{SD}\right)$.

\begin{tabular}{|c|c|c|c|c|c|c|c|c|c|c|}
\hline Sample & Method & Caffa & $p$-Cou & Fer & Chlor & Kaem & Quer & Myr & Naring & Rut \\
\hline \multirow[t]{3}{*}{ “Canario" } & MEKC & b & & & $8.74 \pm 0.31^{c}$ & & & & $6.15 \pm 0.24$ & $7.63 \pm 0.05^{c}$ \\
\hline & HPLC & $1.18 \pm 0.01$ & $0.86 \pm 0.01$ & $1.06 \pm 0.01$ & $9.47 \pm 0.01$ & & & & $8.07 \pm 0.06$ & $5.43 \pm 0.03$ \\
\hline & RSD (\%) & - & - & - & 6 & - & - & - & 19 & 24 \\
\hline \multirow[t]{3}{*}{ “Pera” } & MEKC & & & & $5.89 \pm 0.21^{c}$ & & & & $3.07 \pm 0.12$ & $27.37 \pm 0.00$ \\
\hline & HPLC & $0.96 \pm 0.01$ & & & $6.85 \pm 0.22$ & & & & $3.59 \pm 0.10$ & $21.43 \pm 0.16$ \\
\hline & RSD (\%) & - & - & - & 11 & - & - & - & 11 & 17 \\
\hline \multirow[t]{3}{*}{ "Cherry" } & MEKC & & & $1.59 \pm 0.06 \mathrm{c}$ & $23.51 \pm 1.03$ & & & $3.35 \pm 0.08^{c}$ & $4.20 \pm 0.12$ & $30.92 \pm 1.36$ \\
\hline & HPLC & $1.54 \pm 0.01$ & $1.55 \pm 0.00$ & $1.31 \pm 0.03$ & $19.56 \pm 0.08$ & & & $2.62 \pm 0.03$ & $3.81 \pm 0.03$ & $24.98 \pm 0.24$ \\
\hline & RSD (\%) & - & - & 14 & 13 & - & - & 17 & 7 & 15 \\
\hline
\end{tabular}




\begin{tabular}{|c|c|c|c|c|c|c|c|c|c|c|}
\hline \multirow[t]{3}{*}{ “Kalvert" } & MEKC & & & & $17.86 \pm 0.31$ & & & & $7.93 \pm 0.31$ & $43.58 \pm 0.41$ \\
\hline & HPLC & $1.34 \pm 0.03$ & $0.86 \pm 0.00$ & $0.80 \pm 0.02$ & $15.62 \pm 0.36$ & & & & $9.99 \pm 0.17$ & $35.00 \pm 0.03$ \\
\hline & RSD (\%) & - & - & - & 9 & - & - & - & 16 & 15 \\
\hline \multirow[t]{3}{*}{ “Beef” } & MEKC & & & & $5.25 \pm 0.10^{c}$ & & & & & $4.07 \pm 0.22^{c}$ \\
\hline & HPLC & $0.94 \pm 0.02$ & & $0.86 \pm 0.01$ & $5.68 \pm 0.11$ & & & & & $3.11 \pm 0.08$ \\
\hline & RSD (\%) & - & - & - & 6 & - & - & - & - & 19 \\
\hline \multirow[t]{3}{*}{ S. pimpinellifolium } & MEKC & $3.81 \pm 0.61 c$ & & $3.57 \pm 0.20 c$ & $264.21 \pm 2.08$ & & & $3.70 \pm 0.02^{c}$ & $5.70 \pm 0.12$ & $33.61 \pm 0.41$ \\
\hline & HPLC & $4.43 \pm 0.20$ & $1.32 \pm 0.04$ & $2.78 \pm 0.07$ & $307.07 \pm 1.30$ & & & $2.67 \pm 0.09$ & $4.80 \pm 0.01$ & $32.18 \pm 0.06$ \\
\hline & RSD (\%) & 11 & - & 18 & 11 & - & - & 23 & 12 & 3 \\
\hline \multirow[t]{3}{*}{ S. neorickii } & MEKC & $5.21 \pm 0.03^{c}$ & $9.68 \pm 0.48$ & $1.97 \pm 0.13$ & $152.02 \pm 1.65$ & & & $5.34 \pm 0.14^{c}$ & & $92.47 \pm 2.25$ \\
\hline & HPLC & $5.86 \pm 0.11$ & $7.90 \pm 0.17$ & $2.16 \pm 0.00$ & $191.32 \pm 1.96$ & $1.81 \pm 0.03$ & & $5.84 \pm 0.09$ & & $93.21 \pm 0.80$ \\
\hline & RSD (\%) & 8 & 3 & 7 & 16 & - & - & 6 & - & 1 \\
\hline
\end{tabular}

a Caffeic acid (caff), p-coumaric acid ( $p$-cou), ferulic acid (fer), chlorogenic acid (chlor), kaempferol (kaem), quercetin (quer), myricetin (myr), naringenin (naring), rutin (rut). b

${ }^{\mathbf{c}}$ Under Limit of Quantification. Values included to test the capabilities of the method under low concentrations and only to provide a comparison with HPLC determination.

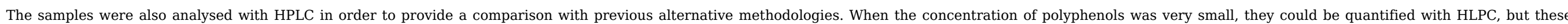

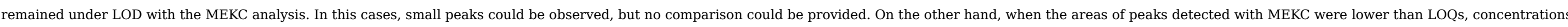

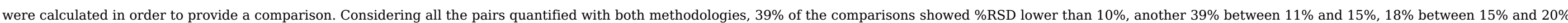
and a $4 \%$ between $20 \%$ and $24 \%$. In $40 \%$ of the determinations with $\%$ RSD higher than $15 \%$ the quantification was made under LOQ, and thus a higher $\%$ RSD was expected.

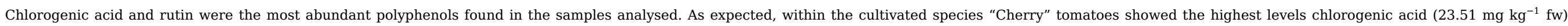

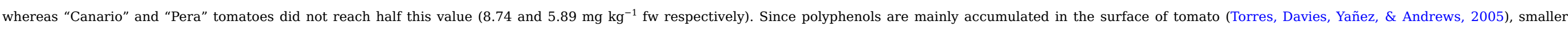

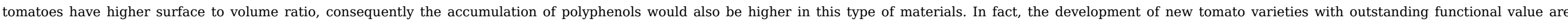

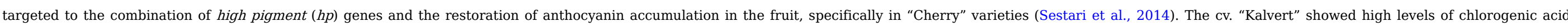

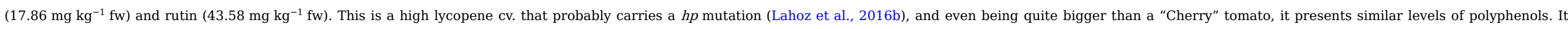

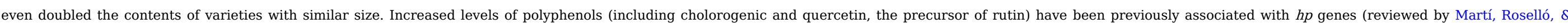
Cebolla-Cornejo, 2016), thus confirming that this cv. might carry an $h p 1$ or $h p 2$ mutation.

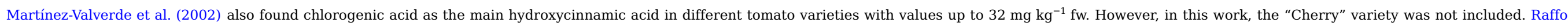

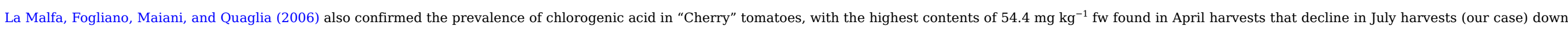
to $31.2 \mathrm{mg} \mathrm{kg}^{-1} \mathrm{fw}$.

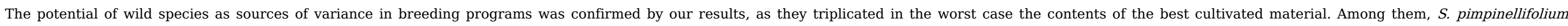

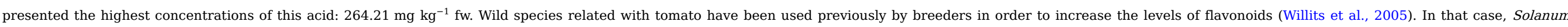

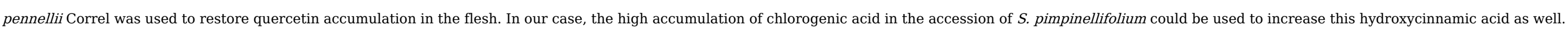




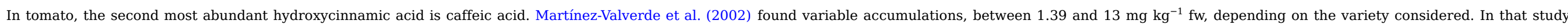

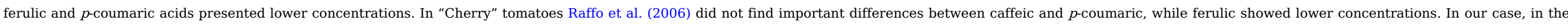

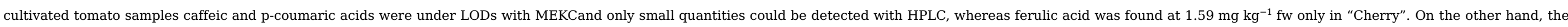
highest quantities of hydroxycinnamic acids were found in S. neorickii.

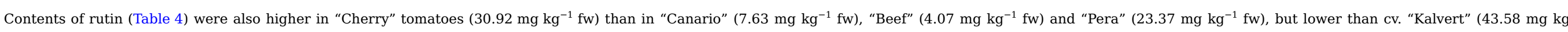

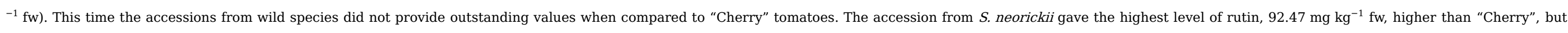
maybe not enough to justify its use in breeding programs.

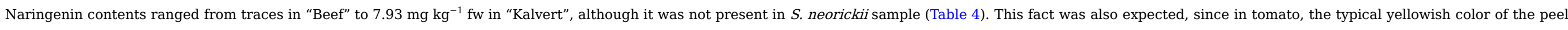
is due to the accumulation of naringenin chalcone (Ballester et al., 2010), and this trait is not present in the green fruit of $S$. neorickii.

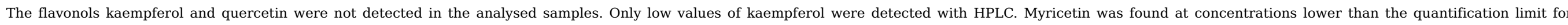

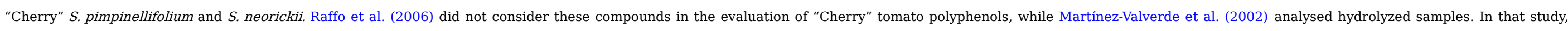

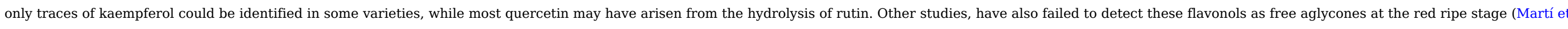
al., 2015).

\title{
4 Conclusion
}

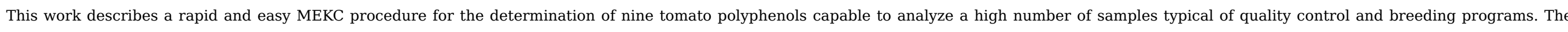

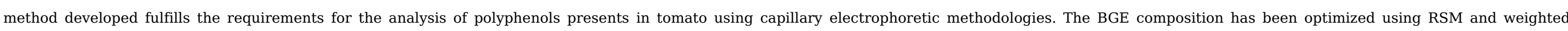

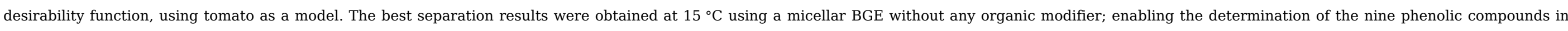

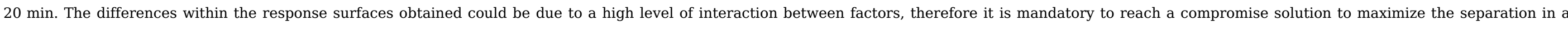
global perspective.

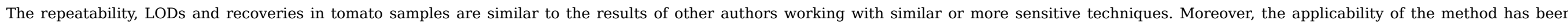

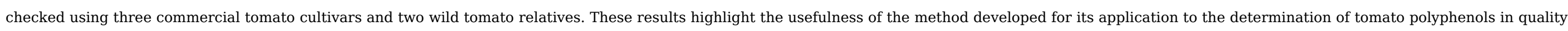
controls or in the development of breeding programs.

\section{Conflict of interest}

\author{
The authors declare no conflict of interest.
}

\section{Acknowledgements}

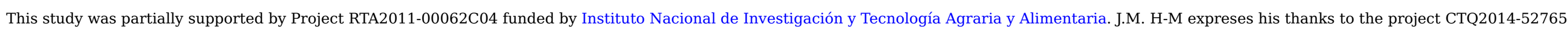
R (MINECO of Spain and FEDER).

\section{Appendix A. Supplementary data}

Supplementary data associated with this article can be found, in the online version, at http://dx.doi.org/10.1016/j.foodchem.2016.10.105

\section{References}

Anderson M.J. and Withcomb P.J., RSM simplified. Optimizing processes using response surface methods for design of experiments, 2005, CRC Press; Boca Raton, FL, USA.

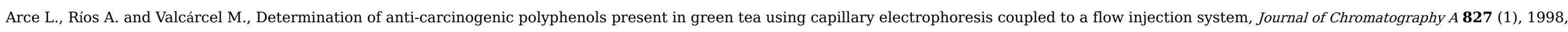

113-120. 


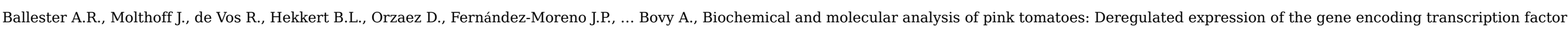
SlMYB12 leads to pink tomato fruit color, Plant Physiology 152, 2010, 71-84.

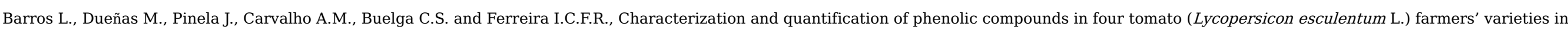
Northeastern Portugal homegardens, Plant Foods for Human Nutrition 67 (3), 2012, 229-234.

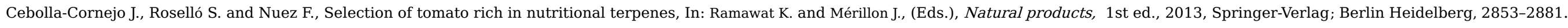

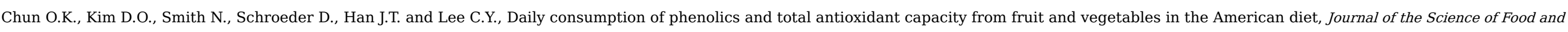
Agriculture 85 (10), 2005, 1715-1724.

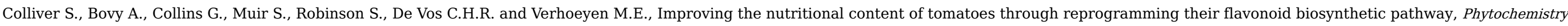
Reviews 1 (1), 2002, 113-123.

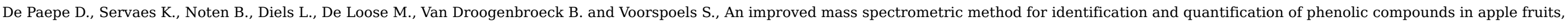
Food Chemistry 136 (2), 2013, 368-375.

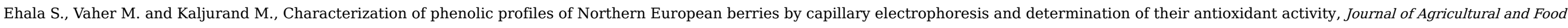
Chemistry 53 (16), 2005, 6484-6490.

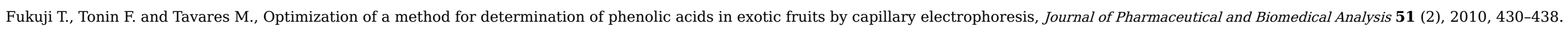

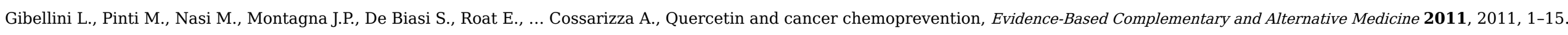

Goldman I.L., Molecular breeding of healthy vegetables, EMBO Reports 12 (2), 2011, 96-102.

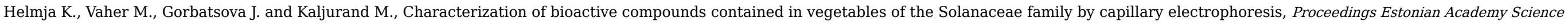
Chemistry 56 (4), 2007, 172-186.

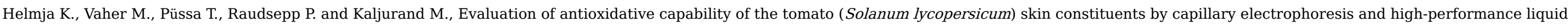
chromatography, Electrophoresis 29 (19), 2008, 3980-3988.

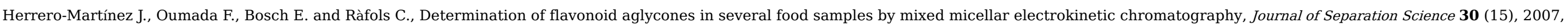
2493-2500.

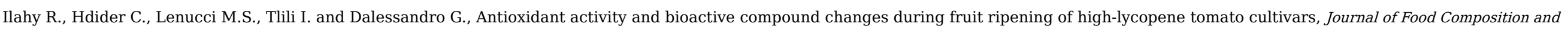
Analysis 24 (4-5), 2011, 588-595.

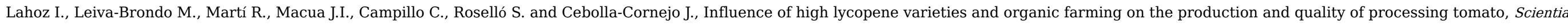
Horticulturae 204, 2016b, 128-137.

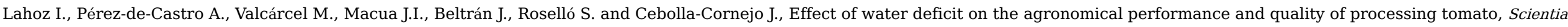
Horticulturae 200, 2016a, 55-65.

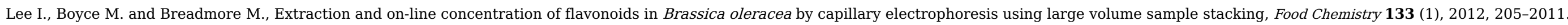

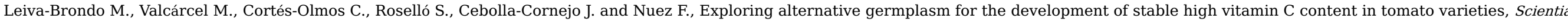
Horticulturae 133, 2012, 84-88.

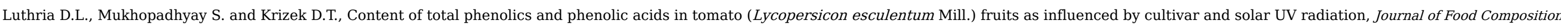


and Analysis 19 (8), 2006, 771-777.

Martí R., Roselló S. and Cebolla-Cornejo J., Tomato as a source of carotenoids and polyphenols targeted to cancer prevention, Cancers 8 (6), 2016, 58.

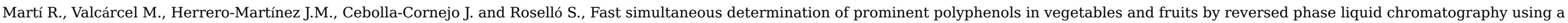
fused-core column, Food Chemistry 169, 2015, 169-179.

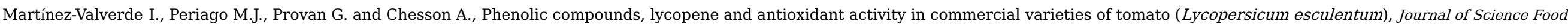
Agriculture 82 (3), 2002, 323-330.

Mes P.J., Boches P., Myers J.R. and Durst R., Characterization of tomatoes expressing anthocyanin in the fruit, Journal of the American Society for Horticultural Science 133 (2), $2008,262-269$.

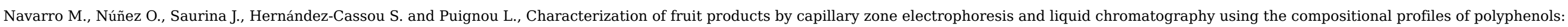
Application to authentication of natural extracts, Journal of Agricultural and Food Chemistry 62 (5), 2014, 1038-1046.

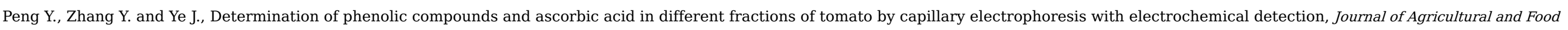
Chemistry 56 (6), 2008, 1838-1844.

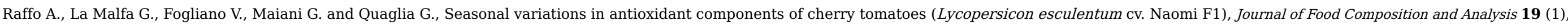
2006, 11-19.

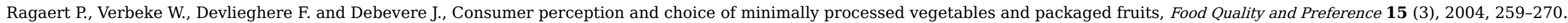

Ramos S., Cancer chemoprevention and chemotherapy: Dietary polyphenols and signalling pathways, Molecular Nutrition \& Food Research 52 (5), $2008,507-526$.

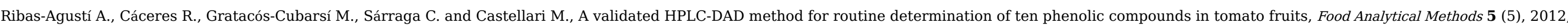
1137-1144.

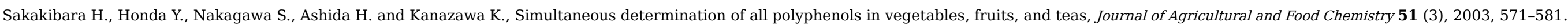

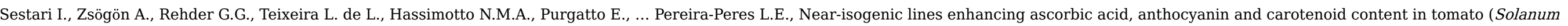
lycopersicum L. cv Micro-Tom) as a tool to produce nutrient-rich fruits, Scientia Horticulturae 175, 2014, 111-120.

Shen Y.C., Chen S.L. and Wang C.K., Contribution of tomato phenolics to antioxidation and down-regulation of blood lipids, Journal of Agricultural and Food Chemistry 55 (16), 2007 , 6475-6481.

Siró I., Kápolna E., Kápolna B. and Lugasi A., Functional food. Product development, marketing and consumer acceptance - a review, Appetite 51 (3), $2008,456-467$.

Slimestad R., Fossen T. and Verheul M.J., The flavonoids of tomatoes, Journal of Agricultural and Food Chemistry 56 (7), $2008,2436-2441$.

Stagos D., Amoutzias G.D., Matakos A., Spyrou A., Tsatsakis A.M. and Kouretas D., Chemoprevention of liver cancer by plant polyphenols, Food and Chemical Toxicology 50 (6), 2012, 2155-2170.

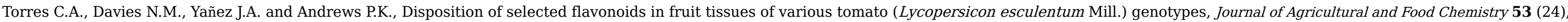
2005, 9536-9543.

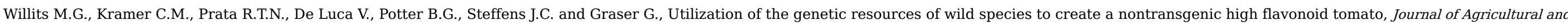
Food Chemistry 53 (4), 2005, 1231-1236.

\section{Appendix A. Supplementary data}


Highlights

- MEKC-DAD method for the determination of nine tomato polyphenols in 20 min.

- BGE used consisted in a $11.3 \mathrm{mM}$ borax buffer containing $11.2 \mathrm{mM}$ SDS adjusted to $\mathrm{pH} 8.5$.

- Separation of polyphenols was optimized using RSM and weighted desirability function.

- It was applicable to high throughput analysis typical from breeding programs.

\section{Queries and Answers}

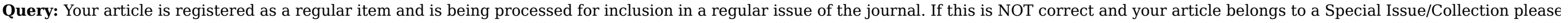
contact r.pio@elsevier.com immediately prior to returning your corrections.

Answer: It's correct, the article is for inclusion in a regular issue of the journal.

Query: The author names have been tagged as given names and surnames (surnames are highlighted in teal color). Please confirm if they have been identified correctly.

Answer: They are correct.

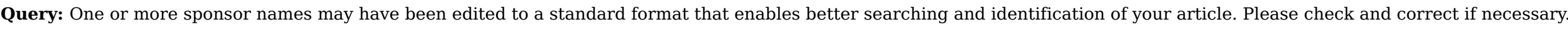

Answer: They are correct.

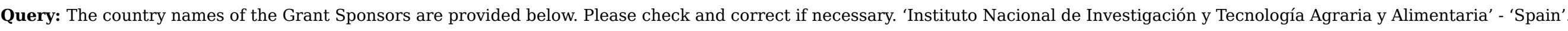

Answer: They are correct. 